\title{
Accuracy of Field Assessment Stroke Triage for Emergency Destination for Diagnosis of Acute Ischemic Stroke Patients
}

\author{
(1) Mohammad Nasr-Esfahani ${ }^{1}$, (1) Farhad Heydari ${ }^{1}$, (1) Elham Izadi-Dastgerdi², (1) Azade Fereidouni Golsefidi², \\ (1) Pegah Noorshargh ${ }^{3}$
}

1Department of Emergency Medicine, Isfahan University of Medical Sciences, Faculty of Medicine, Isfahan, Iran

2Faculty of Nursing and Midwifery, Isfahan University of Medical Science, Isfahan, Iran

${ }^{3}$ Young Researchers and Elite Club, Isfahan (Khorasgan) Branch, Islamic Azad University, Isfahan, Iran

\begin{abstract}
Aim: Acute stroke is one of the most common and debilitating diseases. Rapid diagnostic measures undertaken upon hospital admission and reduction of the treatment duration will increase access to treatment. The purpose of this study is to examine the accuracy of Field Assessment Stroke Triage for Emergency Destination (FAST-ED) in terms of stroke diagnosis.

Materials and Methods: This prospective diagnostic accuracy study was conducted between March 2019 and January 2020. All adult patients transferred to the ED and suspected of having an acute ischemic stroke who had undergone brain magnetic resonance imaging (MRI) were eligible for inclusion. After data collection, receiver operating characteristic curve analysis was performed, and sensitivity, specificity, positive predictive value, and negative predictive value of FAST-ED were calculated and compared with those of the National Institute of Health Stroke Scale (NIHSS) scale.

Results: A total of 314 patients who had received MRI within the first 24 hours of symptom onset were included in this study. The mean age was 67.95 \pm 13.11 years, and 184 patients (58.60\%) were male. Of all patients with suspicion of ischemic stroke, 274 (87.26\%) were diagnosed on the basis of the gold standard. The best predictor of stroke in FAST-ED, with a sensitivity of 0.880 and specificity of 0.575 , was a cut-off point of 2 (area under the curve: 0.836). The distribution of all FAST-ED symptoms was significant in the stroke and non-stroke groups, with the exception of "Eye deviation."
\end{abstract}

Conclusion: The FAST-ED scale is relatively simple and has a comparable ability to recognize AIS to that of the more complex NIHSS.

Keywords: Stroke, triage, acute cerebral ischemia, clinical prediction rules, emergency care

\section{Introduction}

Acute stroke is one of the most common and debilitating diseases (1). Despite major advances in recent years, however, stroke has become one of the leading causes of death worldwide. Stroke was the cause of one in 15 deaths in the United States, and 50\% of these deaths occurred outside the hospital (2).

Previous studies have reported an incorrect stroke diagnosis (false positives) of 30-40\% and a misdiagnosis of stroke (false negatives) of $2-26 \%$ (3). The post-ictal phase of seizures, hypoglycemia, etc., is the differential diagnosis of strokes and taking a complete history, careful physical examination, and the use of diagnostic tools can help differentiate them $(3,4)$.

The main predictor of recovery in cases of acute ischemic stroke is the time elapsed between the onset of symptoms and reperfusion therapy. Rapid triage and rapid transport are critical for patients with acute stroke in a pre-hospital setting. Rapid diagnostic measures after entering the hospital and shortening the duration of treatment will increase the chances of access to treatment 
$(5,6)$. Rapid treatment with intravenous treatment is effective for patients with an ischemic stroke of $<4.5$ hours after onset (5-7).

The National Institute of Health Stroke Scale (NIHSS) can comprehensively assess the degree of neurological impairment in stroke patients. However, NIHSS is complex and timeconsuming (8).

Some existing simplified stroke screens, such as Melbourne Ambulance Stroke Screen, the Face Arm Speech Test (FAST), Cincinnati Prehospital Stroke Severity Scale (CPSSS) and its modification and Recognition of Stroke in the Emergency Room (ROSIER) were designed to detect strokes $(1,8,9)$. The accuracy of these clinical scales varies between 0.75 and 0.80 and differs slightly from each other (8-10). Other prehospital stroke scales have been proposed to identify patients experiencing an acute stroke. Choosing a scale depends on both its accuracy and ease of use. An increasing number of studies assessing the diagnostic performance of clinical assessment tools have been seen in recent years. The accuracy and effectiveness of a new criterion that is comparable in sensitivity, specificity, etc. to other prehospital scales is important and plays an important role in the rapid and accurate diagnosis of acute stroke and thus increasing the patient's chances of successful treatment.

Therefore, we conducted a prospective cohort study to examined the Field Assessment Stroke Triage for Emergency Destination (FAST-ED) score and compared it to NIHSS for detecting acute stroke.

\section{Materials and Methods}

This prospective diagnostic accuracy study had been conducted between March 2019 to January 2020 at at Al-Zahra and Kashani Hospitals, two university hospitals in Isfahan, Iran. The study protocol has been approved by the Ethics Committee of Isfahan University of Medical Sciences (Code: IR.MUI.MED.REC.1397.336)

The FAST-ED Scale [facial palsy (scored 0-1), arm weakness (0-2), speech changes (0-2), time [documentation for decision making but no points], eye deviation (0-2), and denial/neglect (0-2)] was designed based on items of the NIHSS (Table 1).

We enrolled consecutive patients transferred to the emergency department (ED) and suspected of having an acute ischemic stroke. Those who did not receive magnetic resonance imaging (MRI) in the first 24 hours of symptom onset before intravenous thrombolysis or endovascular therapy were excluded.

Patients were excluded if there was evidence of head trauma, previous stroke, known neurological disease, or previous neurological surgery.
A checklist will be used to collect information. For this purpose, a checklist consisting of three sections will be prepared. The first part of the checklist is related to basic information and demographics data of patients, including age, gender, past medical history (hypertension, diabetes, dyslipidemia, ischemic heart disease, seizure, coagulopathy, cerebral infarction, and TIA), NIHSS Score, and time from onset of symptoms, the second part includes five items related to FAST-ED Criteria based on physical examination, and the third part includes the final diagnosis of patients. It was based on the results of the MRI, which was considered the gold standard in this study. The brain MRI was reported by radiologists.

In the present study, all patients transferred to the emergency department who have symptoms of stroke, or are suspected of having a stroke, will be evaluated by an emergency physician (emergency medicine resident, or specialist) and the FAST-ED Criteria and five items will be completed and registered in the checklist.

\section{Statistical Analysis}

SPSS v.22 software was used for statistical analysis. Continuous variables are presented as mean \pm standard deviation. Categorical variables were reported as number and percentage.

Comparisons of variables between two groups (with and without stroke) were conducted by the chi-square test for categorical variables and the independent t-test for continuous variables. We used the Mann-Whitney $U$ test to compare of median \pm Interquartile Range Score of FAST-ED in patients with and without stroke.

Sensitivity and specificity with 95\% confidence interval (CI), positive and negative predictive value, positive and negative likelihood ratio were calculated using several different thresholds of the FAST-ED. The discriminatory performances of FAST-ED were assessed by analysing the Receiver Operating Characteristic (ROC) curve. We calculated the area under the ROC curve (AUC) to distinguish between FAST-ED and NIHHS between two groups (with and without stroke). The Youden Index was used to evaluate the optimal threshold of the FAST-ED scale. A cut-off of 6, 7, and 10 points in the NIHSS was used for comparison.

P-value $<0.05$ was considered as statistically significant.

\section{Results}

A total of 363 patients suspected stroke were collected and 49 patients were excluded according to the exclusion criteria. Finally, 314 patients who receive MRI in the first 24 hours of symptom onset and before reperfusion therapy were included. 
The mean age was 67.95 \pm 13.11 years (between 11 to 93 old years) and 184 patients (58.60\%) were males. Of all suspected patients, 274 patients (87.26\%) had ischemic stroke based-on gold standard diagnosis. The mean age in the stroke group was higher than the non-stroke group (69.05 \pm 13.62 vs $60.78 \pm 13.63$; $p<0.001)$. There was no significant difference in the past medical history (hypertension, diabetes, dyslipidemia, ischemic heart disease, seizure, coagulopathy, cerebral infarction, and TIA) between the two groups (Table 2).

The median of FAST-ED in with stroke group was significantly higher than the non-stroke group (4.0 vs 2.0; $p=0.001$ ). Of FASTED symptoms, "arm weakness" was highest (82.8\%), and "eye deviation" was the lowest (22.6\%) positive symptoms in the stroke group, respectively.

Except for "eye deviation", other FAST-ED criteria were present in more than half of the stroke patients.

Distribution of all FAST-ED symptoms was significant in stroke and non-stroke group except "eye deviation" $(p<0.05)$ (Table 3).

The sensitivity, specificity, positive predictive value, negative predictive value, negative likelihood ratio, and positive likelihood ratio for different cut-off values of the FAST-ED scale for prediction of acute ischemic stroke (AIS) are shown in Figure 1. Better performance of FAST-ED could be shown at two distinct thresholds of $\geq 2$ and $\geq 3$ (Table 3 ). A FAST-ED scale $\geq 2$ and $\geq 3$ showed sensitivity of 0.88 and 0.77 , specificity of 0.58 and 0.68 , positive predictive value (PPV) of 0.88 and 0.89 , and negative predictive value (NPV) of 0.58 and 0.45 in predicting AIS versus NIHSS $\geq 60.80,0.60,0.88$, and 0.46 and NIHSS $\geq 100.78,0.58$, 0.88 , and 0.38 , respectively (Tables 4 and 5)

ROC curves of the FAST-ED scale with the NIHSS are shown in Figure 1.

\section{Discussion}

It is generally accepted that any patient with clinical signs of stroke needs to be an assessment at a stroke center with advanced imaging to make the best treatment decision for the patient. Rapid triage and rapid transport are very important for patients with acute stroke in a pre-hospital setting. Rapid diagnostic measures after entering the hospital and shortening the duration of treatment will increase the chances of access to treatment.

Most items included in FAST-ED scales except "eye deviation" had a strong correlation with AIS and were easy to assess.

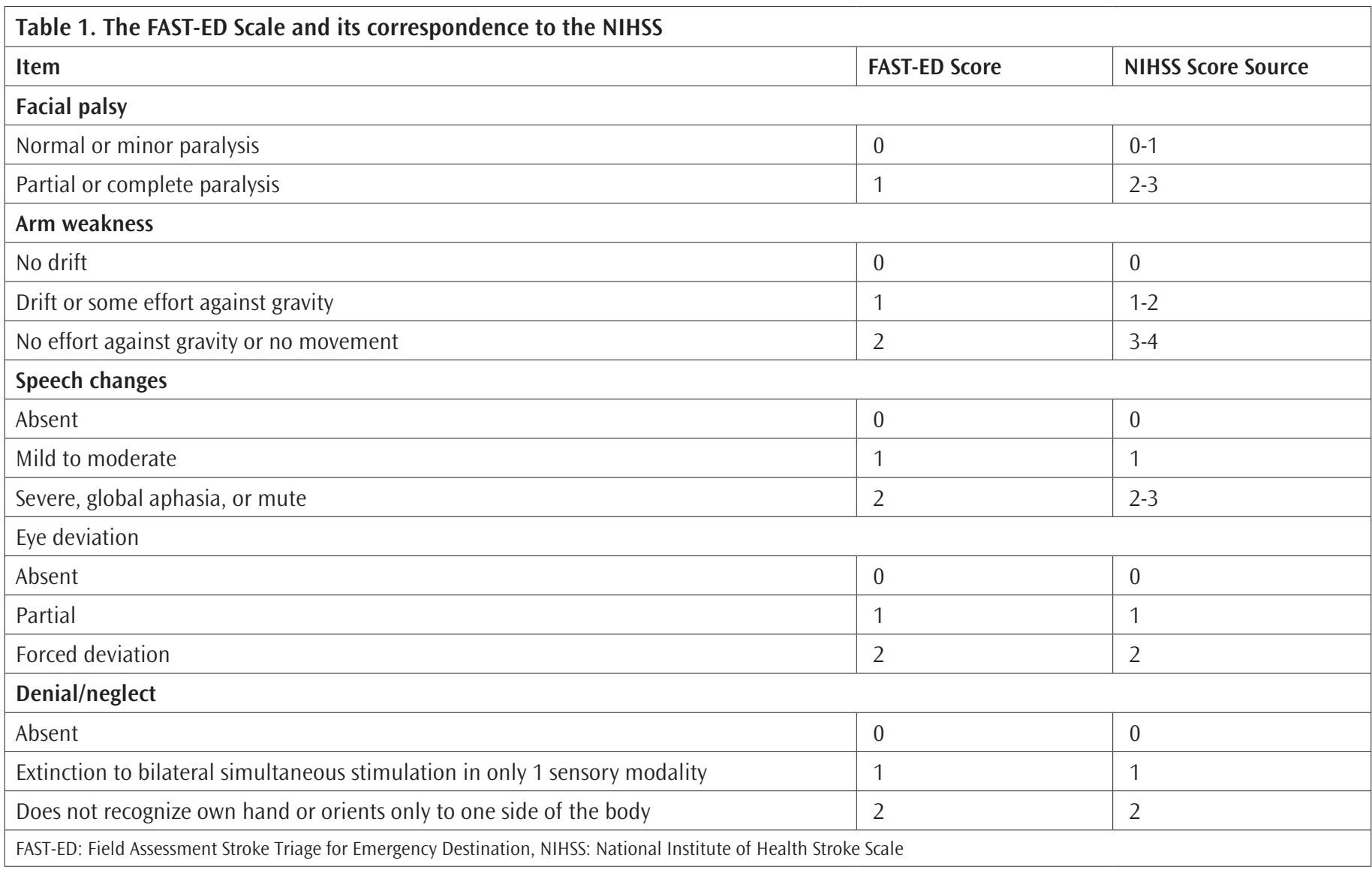




\begin{tabular}{|c|c|c|c|}
\hline & Stroke $(n=274)$ & Non-stroke $(n=40)$ & $p$-value \\
\hline Age; mean (SD), year & $69.05(13.6)$ & $60.78(13.6)$ & $<0.001$ \\
\hline BS; glucometer, mean (SD) & $151.60(65.8)$ & $160.43(100.3)$ & 0.981 \\
\hline Onset to ED mean (SD), hour & 6.68 (19.88) & $3.66(4.75)$ & 0.942 \\
\hline \multicolumn{4}{|l|}{ History of hypertension, n (\%) } \\
\hline Negative & $102(87.9)$ & 14(12.1) & \multirow[t]{2}{*}{0.785} \\
\hline Positive & $172(86.9)$ & $26(13.1)$ & \\
\hline \multicolumn{4}{|l|}{ History of IHD, n (\%) } \\
\hline Negative & $175(86.2)$ & 28(13.8) & \multirow[t]{2}{*}{0.449} \\
\hline Positive & $99(89.2)$ & $12(10.8)$ & \\
\hline \multicolumn{4}{|l|}{ History of smoking, n (\%) } \\
\hline Non-smoker & $246(87.9)$ & $34(12.1)$ & \multirow[t]{2}{*}{0.363} \\
\hline Smoker & $28(82.4)$ & $6(17.6)$ & \\
\hline \multicolumn{4}{|l|}{ DM, n (\%) } \\
\hline Negative & $182(87.9)$ & $25(12.1)$ & \multirow[t]{2}{*}{0.625} \\
\hline Positive & $92(86.0)$ & $15(14.0)$ & \\
\hline \multicolumn{4}{|l|}{ Coagulopathy, n (\%) } \\
\hline Negative & $273(87.2)$ & $40(12.8)$ & \multirow[t]{2}{*}{0.999} \\
\hline Positive & $1(100.0)$ & $0(0.0)$ & \\
\hline \multicolumn{4}{|l|}{ History of stroke, n (\%) } \\
\hline Negative & $236(86.1)$ & 38 (13.9) & \multirow[t]{2}{*}{0.134} \\
\hline Positive & $38(95.0)$ & $2(5.0)$ & \\
\hline \multicolumn{4}{|l|}{ HLP, n (\%) } \\
\hline Negative & 234 (86.7) & $36(13.3)$ & \multirow[t]{2}{*}{0.434} \\
\hline Positive & $40(90.9)$ & $4(9.1)$ & \\
\hline \multicolumn{4}{|l|}{ Seizure, n (\%) } \\
\hline Negative & $270(87.4)$ & 39 (12.6) & \multirow[t]{2}{*}{0.496} \\
\hline Positive & $4(80.0)$ & $1(20.0)$ & \\
\hline
\end{tabular}

In analysing the strength of the FAST-ED scale for our patient population, FAST-ED scale $\geq 2$ and $\geq 3$ showed a sensitivity of 0.88 and 0.77 , a specificity of 0.58 and 0.68 , PPV of 0.88 and 0.89 , and NPV of 0.58 and 0.45 in predicting AIS. This analysis compares with Lima et al.'s (11) and Carr et al.'s (12) study.

Lima et al. (11) assessed that FAST-ED $\geq 4$ had a sensitivity of 0.60 , the specificity of 0.89 , the positive predictive value of 0.72 , and a negative predictive value of 0.82 .

Carr et al. (12) assessed the sensitivity for FAST-ED greater than or equal to 4 as 0.80 , specificity 0.68 , PPV 0.40 , and NPV 0.93 .

Our results were slightly different in which a positive predictive value of $88 \%$ and a negative predictive value of $57.5 \%$ were reported.
In our study, FAST-ED had a high PPV for acute stroke and appeared to be effective for identifying patients who required triage to a stroke center.

FAST-ED scales have also been developed to predict AIS in the prehospital setting and demonstrated good sensitivity and acceptable specificity, it has a sensitivity of 88.0\% [95\% Confidence interval (Cl); $81.5 \%-92.9 \%]$ and a specificity of $57.5 \%(95 \% \mathrm{Cl}$; 40.9\%-72.9\%) in its best cut-off point (score $\geq 2$ ). It demonstrated a comparable ability when compared with the more complex NIHSS score and other similar scales.

Several scales have been developed to be used in pre-hospital and hospital settings to screen stroke patients. There is a significant difference in the diagnostic accuracy of the scales 


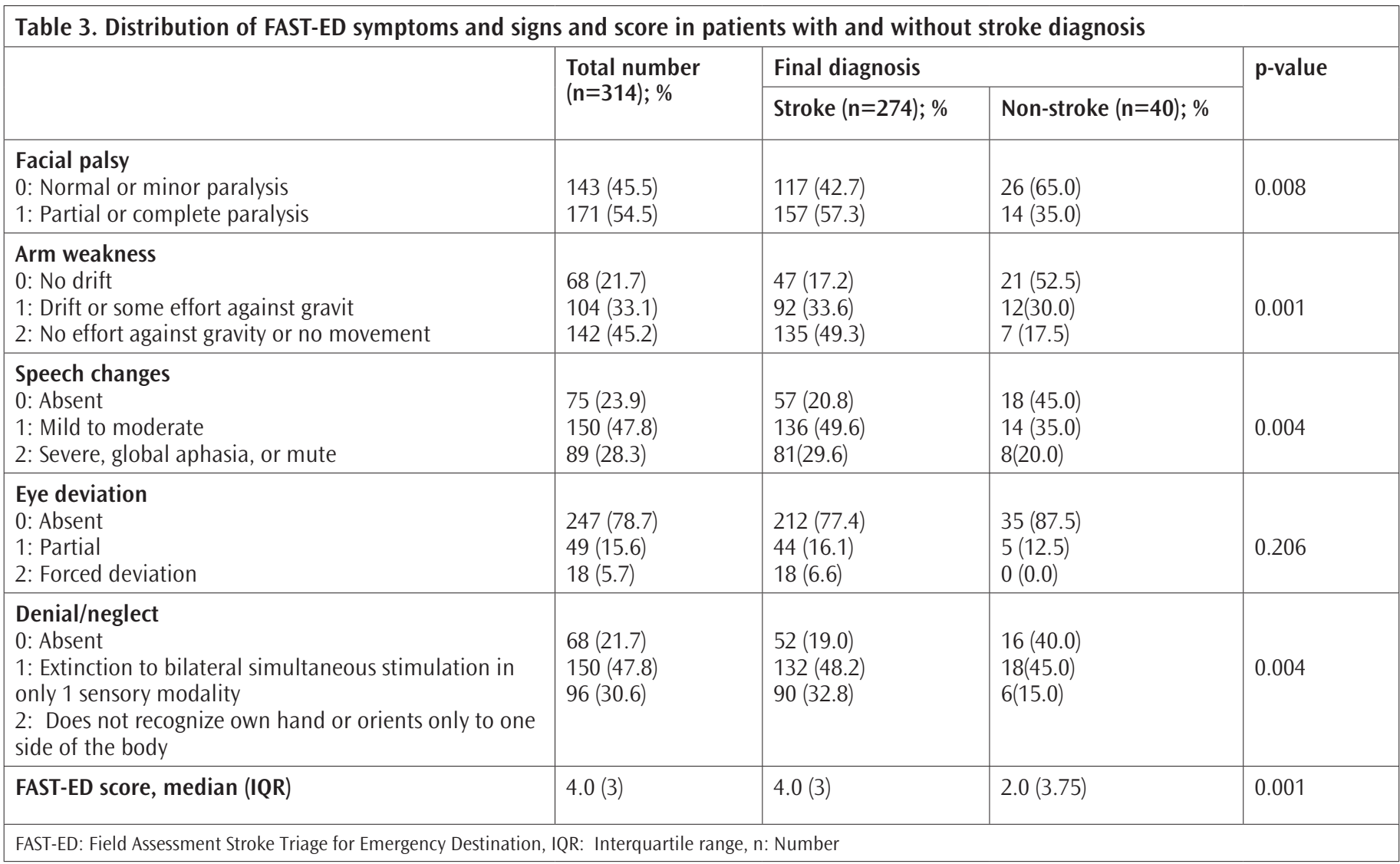

\begin{tabular}{|c|c|c|c|c|c|c|c|}
\hline FAST-ED & Sensitivity (95\% CI) & Specificity $(95 \% \mathrm{CI})$ & LR+ & LR- & PPV & NPV & Youden index \\
\hline$\geq 1$ & $97.2(92.9-99.2)$ & $35.0(20.6-51.7)$ & 1.50 & 0.08 & 84.1 & 77.8 & 0.322 \\
\hline$\geq 2 *$ & $88.0(81.5-92.9)$ & $57.5(40.9-72.9)$ & 2.07 & 0.21 & 88.0 & 57.5 & 0.455 \\
\hline$\geq 3$ & $76.8(68.9-83.4)$ & $67.5(50.9-81.4)$ & 2.36 & 0.34 & 89.3 & 45.0 & 0.443 \\
\hline$\geq 4$ & $57.0(48.5-65.3)$ & $75.0(58.8-87.3)$ & 2.28 & 0.57 & 89.0 & 33.0 & 0.320 \\
\hline$\geq 5$ & $39.4(31.3-48.0)$ & $90.0(76.3-97.1)$ & 3.94 & 0.67 & 93.3 & 29.5 & 0.294 \\
\hline$\geq 6$ & $16.9(11.1-24.1)$ & $95.0(83.0-99.2)$ & 3.38 & 0.87 & 92.3 & 24.4 & 0.119 \\
\hline$\geq 7$ & $6.3(2.9-11.7)$ & $100.0(91.1-100.0)$ & - & 0.94 & 100.0 & 23.1 & 0.060 \\
\hline$\geq 8$ & $0.7(0.1-3.9)$ & $100.0(91.1-100.0)$ & - & 0.99 & 100.0 & 22.1 & 0.070 \\
\hline$\geq 9$ & $0.0(0.0-2.6)$ & $100.0(91.1-100.0)$ & - & 1.00 & 22.0 & - & 0.000 \\
\hline \multicolumn{8}{|c|}{$\begin{array}{l}\text { FAST-ED: Field Assessment Stroke Triage for Emergency Destination, Cl: Confidence interval, LR+: positive likelihood ratio, LR-: Negative likelihood ratio, PPV: Positive predictive } \\
\text { value, NPV: Negative predictive value } \\
\text { *: Best cut-off point }\end{array}$} \\
\hline
\end{tabular}

designed to diagnose AIS. Sensitivity values varied from 44\% [Los Angeles Pre-Hospital Stroke Screen (LAPSS) 1998] to 91\% (NIHSSEMS). Specificity ranged from 27\% [Medical Priority Dispatch System (MPDS)] to 98\% (LAPSS 1998). FABS showed the best diagnostic accuracy values. The FABS tool which was designed specifically for detecting stroke mimics and included additional clinical information, such as atrial fibrillation compared to other well-established tools, for example, ROSIER, demonstrated high sensitivity and specificity rates of about 90\% (PPV: 87\%, NPV: 93\%) (9). 


\begin{tabular}{l|l|l|l|l|l|l|}
\hline Table 5. Comparison of threshold of the FAST-ED and NIHSS according to sensitivity, specificity, PPV and NPV \\
\hline & FAST-ED $\geq \mathbf{2}^{*}$ & FAST-ED $\geq \mathbf{3}$ & FAST-ED $\geq \mathbf{4}$ & NIHSS $\geq \mathbf{6}$ & NIHSS $\geq \mathbf{7}^{*}$ & NIHSS $\geq \mathbf{1 0}$ \\
\hline Sensitivity & 88.0 & 76.8 & 57.0 & 80.3 & 84.1 & 77.8 \\
\hline Specificity & 57.5 & 67.5 & 75.0 & 60.0 & 88.0 & 57.5 \\
\hline PPV & 88.0 & 89.3 & 89.0 & 87.7 & 89.1 & 88.3 \\
\hline NPV & 57.5 & 45.0 & 33.0 & 46.2 & 42.9 & 38.0 \\
\hline $\begin{array}{l}\text { FAST-ED: Field Assessment Stroke Triage for Emergency Destination, NIHSS: National Institute of Health Stroke Scale, PPV: Positive predictive value, NPV: Negative predictive } \\
\text { value } \\
\text { *: Best cut-off point }\end{array}$
\end{tabular}
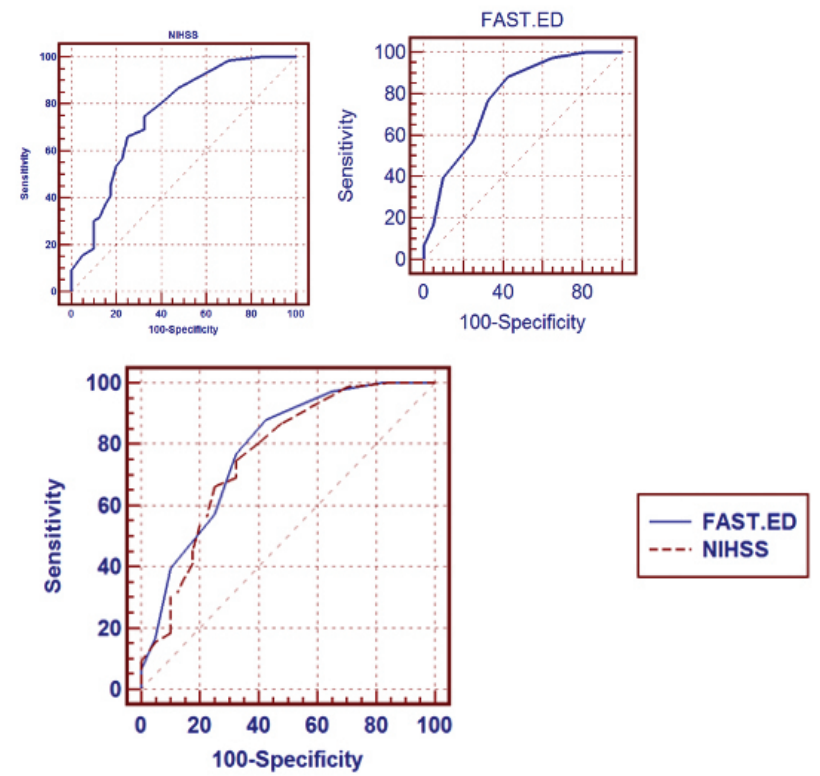

田

\begin{tabular}{|c|c|c|c|c|}
\hline \multirow[b]{2}{*}{ Test Result Variable(s) } & \multirow[b]{2}{*}{ AUC } & \multirow[b]{2}{*}{$P$ value } & \multicolumn{2}{|c|}{ 95\% Confidence Interval } \\
\hline & & & Lower Bound & Upper Bound \\
\hline NIHSS & .821 & .000 & .746 & .895 \\
\hline FAST-ED & .836 & .000 & .759 & .912 \\
\hline
\end{tabular}

Figure 1. The sensitivity, specificity, positive predictive value, negative predictive value, negative likelihood ratio, and positive likelihood ratio for different cut-off values of the FAST-ED scale for prediction of acute ischemic stroke

Smith et al. (5) suggest that the NIHSS is the optimal large vessel occlusion (LVO) prediction instrument in the hospital emergency department, whereas in the prehospital setting, a variety of scales, including the CPSSS, FAST-ED, Los Angeles Motor Scale, and Rapid Arterial Occulsion Evaluation Scale (RACE), could be used without clear evidence for the superiority of 1 scale over the others.

FAST-ED are already familiar with the CPSS and just have added two items to it. Indeed, the FAST-ED scale is simpler than the
RACE scale (six items), which has been validated in the prehospital setting.

In summary, the FAST-ED was designed to be user-friendly and applicable for pre-hospital and hospital settings. FAST-ED $\geq 2$ has promising characteristics in predicting AIS and should be prospectively evaluated to demonstrate clinical use.

\section{Study Limitations}

This study has limitations. First, we did not report the final diagnosis of non-stroke cases. Secondly, we have not compared FAST-ED with all existing prehospital scales.

\section{Conclusion}

FAST-ED scale is relatively simple and has a comparable ability for recognizing AIS to more complex NIHSS. Assessment of both cortical and motor function using FAST-ED or NIHSS demonstrated the good diagnostic accuracy values for selecting subjects with LVO.

\section{Acknowledgements}

The authors would like to express their gratitude to the staff of the ED of Al-Zahra and Kashani Hospital, Isfahan, Iran.

\section{Ethics}

Ethics Committee Approval: The study protocol was approved by the Ethics Committee of Isfahan University of Medical Sciences (IR.MUI.MED.REC.1397.336).

Informed Consent: Before the study, written informed consent was obtained from all parents, before enrolment into the study.

Peer-review: Externally peer-reviewed.

\section{Authorship Contributions}

Surgical and Medical Practices: F.H., M.N.E., E.I.D., A.F.G., Concept: M.N.E., F.H., E.I.D., A.F.G., P.N., Design: M.N.E., F.H., Data Collection or Processing: M.N.E., F.H., E.I.D., A.F.G., Analysis or Interpretation: F.H., P.N., Literature Search: M.N.E., F.H., E.I.D., A.F.G., P.N., Writing: M.N.E., F.H., E.I.D., A.F.G., P.N. 
Conflict of Interest: No conflict of interest was declared by the authors.

Financial Disclosure: The authors declared that this study received no financial support.

\section{References}

1. Loudon W, Wong A, Disney M, Tippett V. Validated pre-hospital stroke scales to predict large vessel occlusion: a systematic review. Australas J Paramedicine. 2019;16.

2. Benjamin EJ, Virani SS, Callaway CW, Chamberlain AM, Chang AR, Cheng S, et al. Heart Disease and Stroke Statistics-2018 Update: A Report From the American Heart Association. Circulation. 2018;137:67-492.

3. Liberman AL, Prabhakaran S. Stroke chameleons and stroke mimics in the emergency department. Curr Neurol Neurosci Rep. 2017;17:15.

4. Yew KS, Cheng E. Acute stroke diagnosis. Am Fam Physician. 2009;80:33-40.

5. Smith EE, Kent DM, Bulsara KR, Leung LY, Lichtman JH, Reeves MJ, et al. Accuracy of prediction instruments for diagnosing large vessel occlusion in individuals with suspected stroke: a systematic review for the 2018 guidelines for the early management of patients with acute ischemic stroke. Stroke. 2018;49:111-22.

6. Ollikainen JP, Janhunen HV, Tynkkynen JA, Mattila KM, Hälinen MM, Oksala NK, et al. The Finnish Prehospital Stroke Scale Detects thrombectomy and thrombolysis candidates-a propensity score-matched study. J Stroke Cerebrovasc Dis. 2018;27:771-7.

7. Venema E, Duvekot MHC, Lingsma HF, Rozeman AD, Moudrous W, Vermeij $\mathrm{FH}$, et al. Prehospital triage of patients with suspected stroke symptoms (PRESTO): protocol of a prospective observational study. BMJ Open. 2019;9:e028810.

8. Li S, Wang A, Zhang X, Wang Y. Design and validation of prehospital acute stroke triage (PAST) scale to predict large vessel occlusion. Atherosclerosis. 2020;306:1-5.

9. Antipova D, Eadie L, Macaden A, Wilson P. Diagnostic accuracy of clinical tools for assessment of acute stroke: a systematic review. BMC Emerg Med. 2019;19:49.

10. Andsberg G, Esbjörnsson M, Olofsson A, Lindgren A, Norrving B, von Euler M. PreHospital Ambulance Stroke Test - pilot study of a novel stroke test. Scand J Trauma Resusc Emerg Med. 2017;25:37.

11. Lima FO, Silva GS, Furie KL, Frankel MR, Lev MH, Camargo ÉC, et al. Field Assessment Stroke Triage for emergency destination: a simple and accurate prehospital scale to detect large vessel occlusion strokes. Stroke. 2016;47:1997-2002.

12. Carr K, Yang Y, Roach A, Shivashankar R, Pasquale D, Serulle Y. Mechanical revascularization in the era of the field assessment stroke triage for emergency destination (FAST-ED): a retrospective cohort assessment in a community stroke practice. J Stroke Cerebrovasc Dis. 2020;29:104472. 